\title{
Involvement of WRN helicase in immortalization and tumorigenesis by the telomeric crisis pathway (Review)
}

\author{
MASANOBU SUGIMOTO ${ }^{1}$, YASUHIRO FURUICHI ${ }^{1}$, TOSHINORI IDE ${ }^{2}$ and MAKOTO GOTO ${ }^{3}$ \\ ${ }^{1}$ GeneCare Research Institute Co., Ltd., Kanagawa 247-0063; ${ }^{2}$ Ehime Prefectural University of Health Sciences, \\ Ehime 791-2101; ${ }^{3}$ Department of Clinical Engineering, Faculty of Medical Engineering, \\ Division of Longevity Science and Anti-Aging Research, Toin University of Yokohama, Yokohama 225-0025, Japan
}

Received January 10, 2011; Accepted April 21, 2011

DOI: $10.3892 / \mathrm{ol} .2011 .298$

\begin{abstract}
The repeated replication of cells shortens telomeres, culminating in their instability, after which most cells cease to replicate and die. However, a small fraction of the cells become immortalized by maintaining telomeres with activated telomerase activity. It has been proposed that WRN helicase encoded by the WRN gene, the causative gene of Werner syndrome (WS), is required for immortalization by the telomeric crisis pathway (TCP) in a system that uses lymphoblastoid cell lines transformed by the Epstein-Barr virus. Taken together, these characteristics indicate that WRN helicase is also required for the immortalization of epithelial cells by TCP and consequent carcinogenesis, suggesting that the tumorigenesis of epithelial cells by TCP is suppressed in WS lacking the WRN helicase function. Notably, in WS the pathway of alternative lengthening of telomeres without activation of telomerase activity has been suggested to be involved in immortalization and tumorigenesis. This factor is consistent with the abundance of non-epithelial cancers in WS in that the ratio of epithelial to non-epithelial cancers is approximately 1:1 in WS patients compared to 10:1 in the general population. A hypothetical scheme showing the role of WRN helicase in immortalization by means of the supposed 'breakage-fusionbridge cycle' of chromosomes at telomeric crisis is described.
\end{abstract}

\section{Contents}

1. Introduction

2. Role of $W R N$ gene in immortalization by means of telomere crisis pathway

Correspondence to: Dr Masanobu Sugimoto, GeneCare Research Institute Co., Ltd., 19-2 Kajiwara, Kamakura, Kanagawa 247-0063, Japan

E-mail: sugimoto@kamakuranet.ne.jp

Key words: WRN helicase, immortalization, telomeric crisis, alternative lengthening of telomeres
3. Role of $W R N$ gene in breakage-fusion-bridge cycle at telomere crisis pathway

4. Conclusion

\section{Introduction}

The Werner syndrome (WS) is an autosomal recessive disorder causing symptoms of premature aging (1). The causative gene of WS is the WRN gene encoding for WRN protein, a DNA helicase (2). Another characteristic feature of this disorder is a much higher incidence of rare cancers (3). Non-epithelial tumors, including soft-tissue sarcoma and benign meningioma, are associated with WS, as shown by 124 case reports of neoplasia of WS patients from Japan, and 34 case reports from outside Japan between 1939 and August 1995. Notably, the ratio of epithelial to non-epithelial cancers was approximately 1:1 in WS patients compared to 10:1 in the general population. Two telomere maintenance mechanisms, telomerase activation (4) and alternative lengthening of telomeres (ALT) $(5,6)$ exist. In human tumors of the general population over $80 \%$ of carcinomas maintain telomeres by telomerase activation, whereas various types of sarcomas elongate telomeres by means of ALT in the absence of telomerase activity (7). In tumors in WS patients, however, evidence has shown that the telomerase activation pathway by telomeric crisis is blocked, while the ALT pathway is enhanced (8-10), supporting the high incidence of rare cancers in WS.

2. Role of $W R N$ gene in immortalization by means of telomeric crisis pathway

The repeated replication of cells shortens telomeres, culminating in their instability, after which most cells cease to replicate and die. However, a small fraction of the cells become immortalized by maintaining telomeres with activated telomerase activity, known as the telomeric crisis pathway (TCP) (11-13). We showed that lymphoblastoid cell lines (LCLs) transformed by the Epstein-Barr virus generate immortalized cell lines by means of TCP (13). Among 50 LCLs from non-WS individuals, 5 LCLs (10\%) were immortalized and the remaining 45 LCLs were mortal. None of the 44 LCLs (0\%; P<0.031 
against healthy individuals using the Chi-square test) from WS patients were immortalized. Among 11 LCLs obtained from a family with a tendency towards hereditary type 2 diabetes mellitus, 5 LCLs (45.5\%; $\mathrm{P}<0.004$ against healthy individuals, $\mathrm{P}<0.0001$ against WS patients) were immortalized $(8,14)$. These results indicated that WRN helicase is crucial to immortalization by TCP $(8,9)$. These factors together indicate that WRN helicase is also required for the immortalization of epithelial cells by TCP and consequent carcinogenesis, suggesting that the tumorigenesis of epithelial cells by TCP is suppressed in WS patients lacking the WRN helicase function. Notably, the ALT pathway in the absence of telomerase activity in WS has been indicated to be involved in the immortalization and tumorigenesis of non-epithelial tumors (10). These considerations were consistent with the abovementioned abundance of non-epithelial cancers in WS.

\section{Role of $W R N$ gene in breakage-fusion-bridge cycle at telomeric crisis pathway}

In this review, we present and discuss a hypothetical scheme showing the role of WRN helicase in immortalization by means of the supposed 'breakage-fusion-bridge cycle' of chromosomes at telomeric crisis proposed by Ishikawa (11).

When two chromosomes lose telomeric function by shortening (Fig. 1A and B) they fuse, resulting in a dicentric chromosome (Fig. 1C). When the dicentric chromosome is segregated towards two daughter cells during the $\mathrm{M}$ phase, two spindles from each of the two daughter cells may attach to each of the two centromeres, leading to the chromosome being pulled apart (Fig. 1D). Thus, the newly formed ends of the chromosome are non-telomeric. Abnormal cells with nontelomeric chromosomes probably do not survive unless the non-telomeric end is capped again with telomeres.

The de novo addition of telomeres to non-telomeric ends has been reported (15). For instance, during programmed chromosomal healing, telomerase adds telomeric repeats directly to non-telomeric sequences in the protozoan Tetrahymena, forming de novo telomeres. Therefore, to add a telomere to a non-telomeric end, initial activation of the telomerase is required (Fig. 1D and E). Such activation may occur when the repression of the human telomerase reverse transcriptase ( $h T E R T)$ gene is released. Alu-elements and other humanspecific repetitive sequences exist abundantly around the hTERT gene, probably forming a repressed chromatin structure in human cells. In this regard, it has been indicated that chromosomal translocations allow the promoter of hTERT to escape the repressive chromatin environment at telomeric crisis (reviewed in 16). One characteristic of immortalization by telomere crisis in LCLs is that abnormal chromosomes occur simultaneously with activation of the telomerase, and all 11 immortalized LCLs were found to possess abnormal chromosomes (13). Cells of each immortalized LCL share a set of abnormal chromosomes, indicating a clonal origin of LCL cells. However, abnormal chromosomes are not shared among LCLs. During the telomeric crisis towards immortalization a series of marked changes should occur accompanied by the reorganization of chromosomes. These changes may include epigenetic ones around telomeric regions as observed during the generation of human pluripotent stem cells (17).

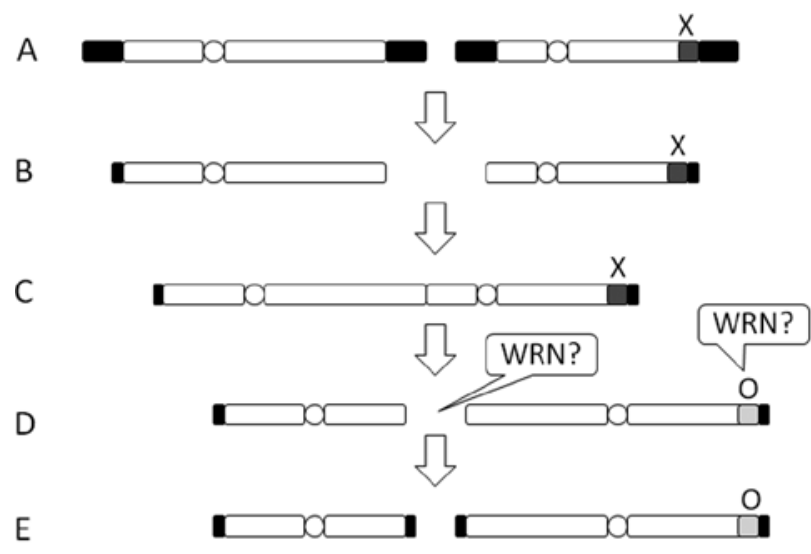

\& $\mathbf{U}$,Telomere; $\square, h$-TERT; X, Off; O, On; WRN, WRN helicase

Figure 1. Hypothetical scheme showing implications of WRN helicase in immortalization by TCP. The image is drawn mainly based on the model of the 'breakage-fusion-bridge' cycle by Ishikawa (11).

Such epigenetic changes may contribute to modification of the hTERT gene region to activate telomerase.

The above-mentioned process of immortalization by TCP is summarized in Fig. 1. WRN helicase may play at least two mutually compatible roles in immortalization by TCP. First, under the supposed epigenetic changes WRN helicase may unwind the repressed state of chromatin DNA that is rich in repetitive elements and resistant to nuclease digestion, leading to modification and activation of the promoter region of the hTERT gene by translocation (reviewed in 16) (Fig. 1D). Second, in the telomerase-mediated de novo addition of telomeres to non-telomeric sequences (Fig. 1E), exonuclease activity of WRN helicase may also be involved in this process to trim the 3 ' end to expose a favorable sequence as a primer for adding a telomere to the non-telomeric end (15). In this regard, a particular sequence, such as GGGAT in the case of human, is predominantly added by telomerase onto the $3^{\prime}$ end of nontelomeric primers. Among the five RecQ helicases, only WRN helicase has exonuclease activity (reviewed in 18), and the fact that the remaining four RecQ helicases cannot complement the function of WRN helicase supports the role of exonuclease activity of WRN helicase in the immortalization by TCP.

\section{Conclusion}

In this review, we proposed a hypothesis regarding the role of WRN helicase in immortalization by means of TCP, indicating that WRN helicase may play an important role in the 'breakage-fusion-bridge cycle'. This hypothesis may lead to future studies at molecular levels in order to elucidate the role of WRN helicase in immortalization and tumorigenesis.

\section{References}

1. Goto M and Miller RW (eds): From Premature Gray Hair to Helicase - Werner Syndrome: Implications for Aging and Cancer. Japan Scientific Societies Press \& Karger, Tokyo, 2001.

2. $\mathrm{Yu} \mathrm{CE}$, Oshima J, Fu YH, et al: Positional cloning of the Werner's syndrome gene. Science 272: 258-262, 1996. 
3. Goto M, Miller RW, Ishikawa Y and Sugano H: Excess of rare cancers in Werner syndrome (adult progeria). Cancer Epidemiol Biomarkers Prev 5: 239-246, 1996.

4. Harley CB, Kim NW, Prowse KR, et al: Telomerase, cell immortality, and cancer. Cold Spring Harb Symp Quant Biol 59: 307-315, 1994.

5. Bryan TM, Englezou A, Gupta J, Bacchetti S and Reddel RR: Telomere elongation in immortal human cells without detectable telomerase activity. Embo J 14: 4240-4248, 1995.

6. Bryan TM and Reddel RR: Telomere dynamics and telomerase activity in in vitro immortalised human cells. Eur J Cancer 33 767-773, 1997.

7. Matsuo T, Shimose S, Kubo T, Fujimori J, Yasunaga Y and Ochi M: Telomeres and telomerase in sarcomas. Anticancer Res 29: 3833-3836, 2009.

8. Sugimoto M, Tahara H, Okubo M, et al: WRN gene and other genetic factors affecting immortalization of human B-lymphoblastoid cell lines transformed by Epstein-Barr virus. Cancer Genet Cytogenet 152: 95-100, 2004.

9. Futami K, Ishikawa $\mathrm{Y}$, Goto M, Furuichi $\mathrm{Y}$ and Sugimoto $\mathrm{M}$ : Role of Werner syndrome gene product helicase in carcinogenesis and in resistance to genotoxins by cancer cells. Cancer Sci 99: 843-848, 2008.

10. Laud PR, Multani AS, Bailey SM, et al: Elevated telomeretelomere recombination in WRN-deficient, telomere dysfunctional cells promotes escape from senescence and engagement of the ALT pathway. Genes Dev 19: 2560-2570, 2005.
11. Ishikawa F: Telomere crisis, the driving force in cancer cell evolution. Biochem Biophys Res Commun 230: 1-6, 1997.

12. Shay JW and Wright WE: Senescence and immortalization: role of telomeres and telomerase. Carcinogenesis 26: 867-874, 2005.

13. Sugimoto M, Tahara H, Ide T and Furuichi Y: Steps involved in immortalization and tumorigenesis in human B-lymphoblastoid cell lines transformed by Epstein-Barr virus. Cancer Res 64: 3361-3364, 2004.

14. Okubo M, Tsurukubo Y, Higaki T, et al: Clonal chromosomal aberrations accompanied by strong telomerase activity in immortalization of human B-lymphoblastoid cell lines transformed by Epstein-Barr virus. Cancer Genet Cytogenet 129: 30-34, 2001.

15. Wang $\mathrm{H}$ and Blackburn EH: De novo telomere addition by Tetrahymena telomerase in vitro. Embo J 16: 866-879, 1997.

16. Zhu J, Zhao Y and Wang S: Chromatin and epigenetic regulation of the telomerase reverse transcriptase gene. Protein and Cell 1: 22-32, 2010.

17. Yehezkel S, Rebibo-Sabbah A, Segev Y, et al: Reprogramming of telomeric regions during the generation of human induced pluripotent stem cells and subsequent differentiation into fibroblast-like derivatives. Epigenetics 6: 63-75, 2011.

18. Shimamoto A, Sugimoto M and Furuichi Y: Molecular biology of Werner syndrome. Int J Clin Oncol 9: 288-298, 2004. 